\title{
Protokoll der Mitgliederversammlung der DEGUM Donnerstag, 15. November 2018, 17:45 Uhr
}

\section{Dreiländertreffen in Basel, Congress-Center Basel, Raum Montreal}

Teilnehmer: 193

\section{Eröffnung}

Der Präsident, PD Dr. med. K.-S. Heling, begrüßt die Teilnehmer der Mitgliederversammlung.

\section{TOP 1: Annahme der Tagesordnung}

Die Einladung mit Tagesordnung wurde satzungsgemäß den Mitgliedern postalisch und per Email zugeschickt und auf der Homepage der DEGUM veröffentlicht. K.-S. Heling stellt den Antrag, die Tagesordnung wie veröffentlicht zu bestätigen:

- TOP 1: Annahme der Tagesordnung

- TOP 2: Annahme des Protokolls der Mitgliederversammlung beim 41. Dreiländertreffen am 12.10.2017 in Linz

- TOP 3: Bericht des Vorstands

- TOP 4: Bericht des Schatzmeisters

- TOP 5: Entlastung des Vorstands

- TOP 6: Aufnahme neuer Mitglieder

- TOP 7: Neufassung der Satzung ${ }^{1}$

- TOP 8: EFSUMB - aktuelle Entwicklungen und Beschlussfassung zum Mitgliedsstatus

- TOP 9: Ehrungen

- Preise (Wissenschaft und Promotion)

- Ehrenmitgliedschaften

- TOP 10: Wahlen

- TOP 11: Berichte der Sektionen und Arbeitskreise

- TOP 12: Verschiedenes

1 Ein Vorschlag zur Neufassung der Satzung wie auch eine erläuternde Synopse sind im kompletten Wortlaut unter www.degum.de/ intern veröffentlicht und können bei Bedarf bei der Geschäftsstelle angefordert werden.
Ein Antrag zu TOP 8 wird vom Vorstand zugelassen und dort vorgestellt.

Die Tagesordnung wird einstimmig mit dieser Ergänzung angenommen.

TOP 2: Annahme des

Protokolls der Mitgliederversammlung beim

\section{Dreiländertreffen am}

\subsubsection{7 in Linz}

Das Protokoll war in der Zeitschrift „Ultraschall in der Medizin" (Heft 1/2018) sowie auf der Homepage der DEGUM veröffentlicht.

Das Protokoll wird ohne Änderungen einstimmig angenommen.

\section{TOP 3: Bericht des}

\section{Vorstands}

K.-S. Heling gibt einen Überblick über die Vereinsaktivitäten des vergangenen Jahres:

Zusammenarbeit mit dem Thieme-Verlag:

Aus der DEGUM heraus wurden 2 neue Editoren vorgeschlagen, die von Thieme auch bestätigt wurden: Prof. Dr. med. Rabih Chaoui aus der Sektion Gynäkologie \& Geburtshilfe und Prof. Dr. med. Uwe Walter aus der Sektion Neurologie.

Weiterhin wurde die Anzahl der Treffen erweitert; um eines im Frühjahr im Rahmen einer Vorstandssitzung, bei dem die Möglichkeit für strategische Gespräche gegeben ist. Das Ziel ist weiterhin die Stärkung des
Ultraschalls und eine Erhöhung der Veröffentlichung von wissenschaftlichen Beiträgen zum Ultraschall in der Zeitschrift UiM.

\section{Wissenschaftsförderung}

Zu den Zielsetzungen des letzten Jahres gehörte die weitere Anhebung der Wissenschaftsförderung: Hier ist ein gegenläufiger Trend zu beobachten - es werden weniger Anträge, aber höhere Fördersummen eingereicht. Insgesamt wurde das Niveau stark angehoben. Die Voraussetzungen für die Förderung sind ausführlich auf der Homepage hinterlegt.

\section{Leitlinienarbeit}

In der Geschäftsstelle Berlin arbeitet Frau Weidlich für das Leitlinien-Sekretariat. Als Beauftragter des Vorstands hat Prof. von Kaisenberg die Ausbildung zum AWMF-zertifizierten Leilinien-Berater abgeschlossen und steht allen Sektionen und Arbeitskreisen für Beratungsfragen zur Leitlinienarbeit zur Verfügung. Für diese Arbeit werden zukünftig bis zu $100000 € / J a h r$ in den Haushalt eingestellt.

Um diese Arbeit transparent zu gestalten, wurde im vergangenen Jahr eine SOP entwickelt, auf der Homepage der DEGUM veröffentlicht und auch den Sektionen und Arbeitskreisen bekannt gegeben. Damit sind die Wege der Antragstellung, der Berichtspflicht und der Mittelvergabe eindeutig vorgegeben.

In diesem Zusammenhang bittet der Präsident um Informationen zu Leitlinien, an denen die Sektionen und Arbeitskreise mitarbeiten, um so eine Rückkopplung in die Geschäftsstelle zu gewährleisten. 
K.-S. Heling stellt exemplarisch die zukünftige Zusammenarbeit der DEGUM mit der DGGG (Dt. Ges. für Gynäkologie \& Geburtshilfe) vor. Beide Gesellschaften arbeiten gleichberechtigt bei Leitlinien zusammen, die den Ultraschall zum Thema haben. Neue AWMF-zertifizierte Leitlinien sollten ebenfalls in der UiM publiziert werden.

\section{Einsatz von nichtärztlichem Personal}

Da es immer wieder Anfragen an den Vorstand zum Einsatz von nichtärztlichem Personal gibt, wurde dieses Thema auf der letzten Sitzung des Erweiterten Vorstands im Januar 2018 ebenfalls diskutiert und es wurde beschlossen, eine Umfrage zum Thema unter den Sektionen und Arbeitskreisen durchzuführen.

Ein weiterer Anlass war das Editorial von Prof. Sidhu in 2017 in der UiM.

Es liegt eine Stellungnahme der DEGUM aus 2012 zum Thema vor mit der Aussage, dass Ultraschall eine ärztliche Tätigkeit ist.

Folgende Fragen wurden über die Geschäftsstelle verschickt: Einsatz von nichtärztlichem Personal im Ultraschall

1. Einsatz von nichtärztlichem Personal: ja/ nein?

2. Wenn ja: Was sollen sie leisten?

3. Wenn nein: Warum?

4. Was ist beim Ultraschall eine zwingend ärztliche Tätigkeit?

Bis auf wenige Ausnahmen haben alle Sektionen und Arbeitskreise sehr differenziert geantwortet:

Die überwiegende Mehrheit ist weiterhin der Ansicht, dass Ultraschall eine ärztliche Tätigkeit ist.

Nach einer angeregten Diskussion im Erweiterten Vorstand wurde beschlossen, über die Art der Veröffentlichung der Ergebnisse auf der nächsten Sitzung des Erweiterten Vorstands erneut zu diskutieren.

\section{Geschäftsstelle}

Die Geschäftsstelle unter Leitung der Geschäftsführerin Marion Schapheer-Risse hat an ihren 2 Standorten in Bonn und Berlin insgesamt 7 Mitarbeiterinnen, denen allen an dieser Stelle herzlich für ihre Arbeit gedankt wird.

Ab 2019 wird zusätzlich eine Assistenz der Geschäftsführerin eingestellt.

Die neue Mitgliederdatenbank bietet vielfache Erleichterungen im gesamten Aufgabenspektrum.

Rechtliche Beratung erfolgt durch Herrn RA Dr. Mecking, Berlin, und die steuerrechtliche Beratung durch die Steuerberatungsgesellschaft Baker Tilly.

\section{Ultraschall-Akademie}

Die Ultraschall-Akademie der DEGUM mit der Geschäftsführerin Frau Katrin Beck hat insgesamt 3 Mitarbeiter - alle Entwicklungen dazu wurden ausführlich im Erweiterten Vorstand vorgestellt.

\section{DLT 2019 Mainz}

Die beiden Kongresspräsidenten Prof. Markus Hahn und Dr. Hans Peter Weskott sind zusammen mit der Kongressorganisation Interplan bereits in der intensiven Phase der Vorbereitung. Anfang Januar tagt die Fortbildungskommission - alle Vorschläge für Anwenderseminare und Refresher-Kurse sind unter fortbildungskommission@degum.de einzureichen.

\section{TOP 4: Bericht des Schatzmeisters}

P. Kozlowski stellt den Jahresabschluss 2017 vor, der von der Steuerberatungsgesellschaft Baker Tilly, Berlin, erstellt wurde.

Der Abschluss 2017 unterscheidet sich von denen der Vorjahre, da durch die Neubewertung des Finanzamtes Berlin der Umfang des wirtschaftlichen Zweckbetriebs erweitert wurde und somit eine Änderung der Zuordnungen unumgänglich war.

Unter ausführlichen Erläuterungen der vereinsinternen Geschichte (Gründung der Ultraschall-Akademie Ende 2011, bisherige steuerliche Bewertung des FA Hamburg) begründet der Schatzmeister den Wechsel vom Hamburger zum Berliner Finanzamt und der erstmaligen steuerlichen Bewertung seit der Akademie-Gründung. Der letzte Freistellungsbescheid war bis 2012 erteilt worden.

Die steuerliche Bewertung der Jahre 2013 2015 ist abgeschlossen, für 2016 müssen noch restliche Fragen geklärt werden. Alle daraus folgenden Steuerforderungen wurden bereits beglichen.

Durch die neue Bewertung des FA Berlins (Erweiterung des wirtschaftlichen Zweckbetriebs durch das Plakettengeschäft und die Zertifizierungen) wurden auch die Ausgaben neu zugeordnet: Personal- und Sachkosten mussten für die genannten Jahre neu zusammengestellt werden. Die Bewertung des Dreiländertreffens für 2016 und der zukünftigen Kongresse steht noch aus.

Der Jahresabschluss 2017 verzeichnet als Ergebnis ein kleines Minus. Auch die Situation bei den Rücklagen hat sich leicht entspannt.

Als Verwendung für die zweckgebundenen Rücklagen wird vorgeschlagen: $256000 €$ für die Vereinszeitschrift und $70000 €$ für die Forschungsförderung.

Diesem Vorschlag stimmt die Mitgliederversammlung einstimmig zu.

Das aktuelle Budget für 2018 hat sich z. B. bei den Ausgaben für die Forschungsförderung wesentlich erhöht und folgt damit den satzungsbedingten Verwendungszwecken.

Ebenfalls sind weitere Ausgaben bei der Leitlinienarbeit zu erwarten, die mit bis zu $100000 €$ in den Haushalt für 2019 eingestellt werden.

Eine Erhöhung wird auch bei den Personalkosten erfolgen, da zum 01.01.2019 eine Assistenz für die Geschäftsführung eingestellt werden soll.

\section{TOP 5: Entlastung des Vorstands}

Th. Müller stellt den Antrag auf Entlastung des Vorstands. Der Antrag wird mit 6 Enthaltungen angenommen. 


\section{TOP 6: Aufnahme neuer Mitglieder}

Seit der letzten Mitgliederversammlung in Linz haben 733 Kolleginnen und Kollegen einen schriftlichen Antrag auf Mitgliedschaft gestellt. Der Vorstand hat diesen Anträgen in seinen Sitzungen zugestimmt.

Die Namen dieser durch den Vorstand aufgenommenen Neumitglieder sind den Mitgliedern der DEGUM durch Auslage am Stand der DEGUM bekannt gegeben worden. Widerspruch gegen die Aufnahme eines oder mehrerer dieser Neumitglieder wurde weder vor noch auf der Mitgliederversammlung erhoben.

Die DEGUM hat aktuell 11011 Mitglieder, davon 10909 ordentliche; Kündigungen gab es 406.

Es sind 2971 Mitglieder mit der Stufe 1 zertifiziert, 1577 mit der Stufe 2, 528 sind mit der Stufe 3 zertifiziert und 718 haben den Kursleiterstatus der Stufe 2 oder 3.

In diesem Rahmen wird allen Gutachtern und Mitarbeiterinnen, die für die Zertifizierungen tätig sind, herzlich für ihre Arbeit gedankt.

\section{TOP 7: Neufassung der Satzung}

Im Rahmen der Einladung zur Mitgliederversammlung wurden die Mitglieder über den Vorschlag für die Neufassung der Satzung samt einer erläuternden Synopse informiert. Der komplette Wortlaut stand im internen Bereich der Homepage zur Verfügung und konnte auch über die Geschäftsstelle angefordert werden.

Ein Vorschlag von M. Wüstner wurde bereits in die veröffentlichte Fassung eingearbeitet. Weiterhin liegt ein Antrag vor von Herrn Dr. med. Christian Jenssen, Herrn Dr. med. Matthias Wüstner, Herrn Prof. Dr. Dieter Nürnberg und Herrn Prof. Dr. Christoph Frank Dietrich. Dieser Antrag bezieht sich auf $\S 2.4$ der Neufassung und wurde nach Rücksprache mit Herrn RA Dr. Mecking und den Einreichern folgendermaßen eingereicht:
„Die Gesellschaft fördert auch im Ausland; ihre Tätigkeit dient dabei neben der Verwirklichung ihrer steuerbegünstigten Zwecke auch der Förderung des Ansehens der Bundesrepublik Deutschland im Ausland. Sie pflegt fachliche Verbindungen mit anderen europäischen Ultraschallgesellschaften und ihren Dachverbänden sowie mit anderen internationalen Fachgesellschaften, in denen Ultraschallmethoden einen hohen Stellenwert haben. Die Gesellschaft wirkt so auf internationaler Ebene an der Weiterentwicklung von Wissenschaft, Forschung und Fortbildung im Zusammenhang mit medizinischen Ultraschallanwendungen mit.“

Weitere Vorschläge für Änderungen oder Ergänzungen werden nicht gestellt. Damit wird die Neufassung der Satzung zur Abstimmung gestellt.

Die Neufassung wird mit 1 Enthaltung angenommen.

\section{TOP 8: EFSUMB - aktuelle Entwicklungen und Beschlussfassung zum Mitgliedsstatus}

Im Vorstand wie auch im gemeinsamen Gespräch mit den Vorständen von ÖGUM und SGUM wurde immer wieder und aktuell auch über den Nutzen der EFSUMB für die Gesellschaften diskutiert.

Eine Umfrage der SGUM, die diese 2017 bei ihren Mitgliedern durchgeführt hat, zum Verbleib jeden einzelnen Mitglieds in der EFSUMB ergab, dass sich ca. 500 Mitglieder für einen persönlichen Verbleib ausgesprochen haben. Dieses Ergebnis wurde der EFSUMB mitgeteilt und entsprechend wurde im Jahr 2018 der aktualisierte Beitrag an die EFSUMB überwiesen.

Aufgrund der aktuellen Satzung der EFSUMB kann aber nur eine Gesellschaft Mitglied der EFSUMB sein - Einzelmitgliedschaften sind nicht vorgesehen.

In der Satzung der EFSUMB ist ebenfalls niedergelegt, dass alle Mitgliederdaten an die EFSUMB weiterzuleiten sind - dem kann aus Datenschutzgründen nicht gefolgt wer- den. Auch die Folgen des Brexits sind bisher noch nicht thematisiert worden.

Aus diesen Gründen hat der Vorstand überlegt, ebenfalls die Mitglieder zu befragen und in der Mitgliederversammlung über die Entscheidung der Mitgliedschaft abstimmen zu lassen.

K.-S. berichtet über die sehr lebhaften und kontroversen Diskussionen in der Sitzung des Erweiterten Vorstands.

Von mehreren DEGUM-Mitgliedern wurde folgender Antrag eingereicht:

„Der medizinische Ultraschall lebt in Wissenschaft, Lehre und Praxis vom internationalen Austausch. Der DEGUM kommt aufgrund ihrer Mitgliederstärke und ihres wissenschaftlichen Potenzials eine besondere Verantwortung zur Entwicklung der europäischen Ultraschallfamilie zu. In der Diskussion um die Rolle der DEGUM in der EFSUMB und um die inhaltliche Ausrichtung der EFSUMB ist die Notwendigkeit einer sachlichen und konstruktiven Debatte deutlich geworden.

Der Vorstand wird beauftragt, innerhalb des Erweiterten Vorstands, der Sektionen und der Arbeitskreise eine strukturierte und transparente Meinungsbildung zur internationalen Vernetzung und zur Rolle der DEGUM in der EFSUMB in Gang zu setzen und zu diesem Zweck eine Klausurtagung des Erweiterten Vorstands unter Einbeziehung der DEGUM-Vertreter in Vorstand und Komitees der EFSUMB vorzubereiten.“

In der sich anschließenden Diskussion wird in mehreren Beiträgen deutlich, dass sich die Mitglieder mehr Zeit wünschen, in der über die EFSUMB informiert und diskutiert werden kann.

Abschließend wird über den eingebrachten Antrag abgestimmt:

Der Antrag wird mit überwiegender Mehrheit positiv abgestimmt und der Vorstand mit der Umsetzung beauftragt. 


\section{TOP 9: Ehrungen}

\section{Preise (Wissenschaft und Promotion)}

Den Wissenschaftspreis 2018 erhält PD Dr. med. Alexander Grimm für die Arbeiten:

„Ultrasound pattern sum score, homogenity score and regional nerve enlargement index for differentiation of demyelinating inflammatory and hereditary neuropathies."

„Nerve Ultrasound as follow-up tool in treated multifocal motor neuropathy."

„Nerve Ultrasound predicts treatment response in CIPD - a prospective follow-up.“

Den Promotionspreis 2018 erhält Dr. rer. nat. Frank Wolfram für die Arbeit:

„Untersuchungen zur Ablation des Bronchialkarzinoms am Modell der flüssigkeitsgefüllten Lunge mithilfe therapeutischer Schallfelder"

Beiden Preisträgern wird nach der Präsentation ihrer Arbeit die Urkunde mit Scheck übereicht.

\section{Ehrenmitgliedschaften}

Neuantrag Prof. Dr. Dr. Robert Sader

Prof Dr. med. A. Bozzato, Vorsitzender der Sektion Kopf-Hals, beantragt die Ehrenmitgliedschaft für Prof. Dr. Dr. med. Robert Sader. Der Antrag wurde satzungsgemäß in den Sitzungen des Vorstands sowie des Erweiterten Vorstands einstimmig angenommen.

Die Mitgliederversammlung stimmt diesem Antrag einstimmig zu.

Damit wird die Ehrenmitgliedschaft für Prof. Sader anlässlich des nächsten Dreiländertreffens in Mainz verliehen.

Verleihung der Ehrenmitgliedschaft an Prof. Dr. med. Dieter Nürnberg

Die Ehrenmitgliedschaft der DEGUM wird Herrn Prof. Dr. med. Dieter Nürnberg verliehen. Dem Antrag war anlässlich der Mitgliederversammlung 2017 in Linz zugestimmt worden. Die Laudatio hält Dr. med. Bernd Brentzel-Beyme.
TOP 10: Wahlen

\section{Wahl des Wahlleiters}

Als Wahlleiter wird Dr. med. Jörg Simanowski vorgeschlagen.

Diesem Vorschlag wird einstimmig zugestimmt.

Satzungsgemäß wechselt der Präsident PD Dr. med. Kai-Sven Heling in die Position des Vizepräsidenten, der Vizepräsident Prof. Dr. med. Dirk Becker verlässt den Vorstand und der Neupräsident Prof. Dr. med. Peter Jecker wechselt in die Position des Präsidenten.

Zu wählen sind die Positionen des Neupräsidenten, des Sekretärs, des Schatzmeisters sowie der beiden Beisitzer.

Der Wahlleiter schlägt vor, die Wahlen in offener Abstimmung vorzunehmen - dieser Vorschlag wird von der Mitgliederversammlung ohne Gegenstimme angenommen.

\section{Neupräsident}

Um die Position des Neupräsidenten bewirbt sich Prof. Dr. med. Josef Menzel. Keine weiteren Vorschläge.

Ergebnis:

Prof. Dr. med. Josef Menzel wird in offener Abstimmung mit 1 Enthaltung gewählt.

\section{Schatzmeister}

Um die Position des Schatzmeisters bewirbt sich der bisherige Schatzmeister, Prof. Dr. med. Peter Kozlowski. Keine weiteren Vorschläge.

Ergebnis:

Prof. Dr. med. Peter Kozlowski wird in offener Abstimmung mit 1 Enthaltung gewählt.

\section{Sekretär}

Um die Position des Sekretärs bewirbt sich Prof. Dr. med. Hans Heynemann. Keine weiteren Vorschläge.

Ergebnis:

Prof. Dr. med. Hans Heynemann wird in offener Abstimmung mit 1 Enthaltung gewählt.

\section{Beisitzer}

Prof. Dr. med. Markus Hahn erklärt seine Bereitschaft zur Kandidatur als Beisitzer. Kein weiterer Vorschlag.

\section{Ergebnis:}

Prof. Dr. med. Markus Hahn wird in offener Abstimmung mit 1 Enthaltung gewählt.

Dr. med. Siegfried Krishnabhakdi erklärt ebenfalls seine Bereitschaft zur erneuten Kandidatur als Beisitzer - ebenfalls kein weiter Vorschlag.

Ergebnis:

Dr. med. Siegfried Krishnabhakdi wird in offener Abstimmung mit 1 Enthaltung gewählt.

Alle Kandidaten nehmen die Wahl an.

Der Präsident Prof. Peter Jecker bedankt sich bei dem Vizepräsidenten PD Dr. KaiSven Heling für den hohen persönlichen Einsatz in seiner Präsidentschaft.

\section{TOP 11: Berichte der}

\section{Sektionen und Arbeitskreise}

P. Jecker fragt nach wichtigen Ergebnissen aus den Sektionen und Arbeitskreisen, die noch vorgestellt werden sollen. Keine weitere Wortmeldung.

Die Jahresberichte müssen bis März 2019 in der Geschäftsstelle eingereicht werden.

\section{TOP 12: Verschiedenes}

Keine weiteren Wortmeldungen.

Der Präsident beendet die Sitzung um 19.35 Uhr.

Prof. Dr. med. Peter Jecker, Präsident, Prof. Dr. med. Hans Heynemann, Sekretär

Protokollführerin: Marion SchapheerRisse 